\title{
September epsilon Perseids observed by the Czech Fireball Network
}

\author{
Lukáš Shrbený and Pavel Spurný
}

\begin{abstract}
Astronomical Institute, Academy of Sciences, Ondřejov, Czech Republic
\end{abstract}
e-mail: shrbeny@asu.cas.cz

Received 16 May 2019 / Accepted 4 July 2019

\begin{abstract}
We present 25 photographic fireballs belonging to the September epsilon Perseid (SPE, IAU \#208) meteor shower observed by the Czech part of the European Fireball Network in 2013-2017. Exceptional high activity of bright photographic fireballs was observed in 2013, while a lower activity, but still higher than in other years, was observed in the period of 2015-2017. Physical properties of these SPE fireballs were studied and compared to other meteor showers. Perseids are found to be the closest analog to SPE. Corrected geocentric radiant of the 2013 outburst fireballs was determined for solar longitude $167.20^{\circ}$ and has right ascension $47.67 \pm 0.04^{\circ}$ and declination $39.493 \pm 0.013^{\circ}(\mathrm{J} 2000.0)$. On the basis of determined heliocentric orbits the parent body of the shower is an unknown long-period comet on retrograde orbit with an orbital period of the order of a thousand years.
\end{abstract}

Key words. meteorites, meteors, meteoroids

\section{Introduction}

Activity of fast meteors radiating from Perseus during September was first described by Denning (1878), who used the visual observations of $\mathrm{G}$. Zezioli performed at Bergamo, Italy between 1867 and 1870 to determine the apparent radiant position of $60^{\circ}$ in right ascension (RA) and $+37^{\circ}$ in declination (Dec) and the activity of the shower in the period of September 5-12. The shower was confirmed by the visual observations of Denning (1882) between 1877 and 1880 and the radiant was found at $\mathrm{RA}=61^{\circ}, \mathrm{Dec}=36^{\circ}$ with maximum activity in the period of September 7-8. Hoffmeister (1948) also included September epsilon Perseids (SPE) in his list of permanent showers but the radiant position $\mathrm{RA}=53^{\circ}$, Dec $=41^{\circ}$ was based on observation during only one night in 1936 , in addition to observation made on September 16, which is out of the period of activity observed earlier so this radiant does not have to be reliable. The fact that the SPE shower was not included in the working list of meteor streams from Cook (1973) suggests either weak or zero activity for as long as a decade. Two decades later, Rendtel (1993) searched the IMO database of visual meteors for radiants in the area of the Perseus and Auriga constellations. He found two separate sources of meteors active in September and October: September Perseids (active around midSeptember) and Delta Aurigids (beginning of October). The geocentric radiant of SPE was found at RA $=51.5^{\circ}, \mathrm{Dec}=39.5^{\circ}$ (B1950.0, corresponding to $\mathrm{RA}=52.3^{\circ}, \mathrm{Dec}=39.7^{\circ}$ in $\mathrm{J} 2000.0$ ) for September 12. Mean parameters of the orbit were perihelion distance, $q=0.75 \mathrm{AU}$, eccentricity, $e=0.95$, and inclination, $i=143^{\circ}$ (based on the combination of seven photographically and three graphically reduced meteors during the period of September 7-28). A more precise position of SPE radiant was determined by Porubčan \& Gavajdová (1994) using the International Astronomical Union Meteor Data Center (IAU MDC) database of photographic orbits. The geocentric radiant position of $\mathrm{RA}=47.2^{\circ}$ and $\mathrm{Dec}=38.9^{\circ}$ (B1950.0, corresponding to $\mathrm{RA}=48.0^{\circ}$, Dec $=39.1^{\circ}$ in $\mathrm{J} 2000.0$ ) was based on three photographic fireballs recorded during the period of September 7-13 (a subset of meteors from Rendtel 1993 was used). Also geocentric velocity, $v_{\mathrm{G}}=65.4 \mathrm{kms}^{-1}$ and mean heliocentric orbit were determined from these data. Mean parameters of the orbit were: $q=0.733 \mathrm{AU}, e=1.03 \pm 0.04$, and $i=140.5^{\circ}$ (Porubčan \& Gavajdová 1994). The following parameters are in J2000.0. Jenniskens et al. (2016) published the mean parameters of 85 SPE video meteors observed in 2011 and 2012: $\mathrm{RA}=48.6^{\circ}, \mathrm{Dec}=39.6^{\circ}, v_{\mathrm{G}}=64.8 \mathrm{kms}^{-1}, q=0.718 \mathrm{AU}$, $e=0.979$, and $i=139.9^{\circ}$. They concluded that the unknown parent comet is a Halley-type comet (HTC).

Recently, two major outbursts of SPE shower were observed. The first one occurred on September 9, 2008 at 8h20m $\pm 20 \mathrm{~m} \mathrm{UT}$ (solar longitude $166.91^{\circ}$ ). The apparent radiant was found approximately at $\mathrm{RA}=49.5^{\circ}$ and $\mathrm{Dec}=43.0^{\circ}$ on the basis of single-station video observations (Jenniskens et al. 2008; Molau \& Kac 2008; Rendtel \& Molau 2010). The second outburst of a SPE shower was observed on September 9, 2013. The time of the maximum activity was determined by Rendtel et al. (2014) to be $22 \mathrm{~h} 18 \mathrm{~m} \pm 7 \mathrm{~m} \mathrm{UT}$ (solar longitude $167.20^{\circ}$, single-station video meteors) and by Gajdoš et al. (2014) to be $22 \mathrm{~h} 05 \mathrm{~m} \pm 10 \mathrm{~m} \mathrm{UT}$ (solar longitude $167.19^{\circ}$, double-station video meteors). Rendtel et al. (2014) showed that the second outburst can be predicted on the basis of the radiant position and the time of the 2008 outburst using the model of Lyytinen \& Jenniskens (2003) and assuming that the particles came from one-revolution trail of a long-period comet (LPC). The predicted 2013 outburst peak is then September 9, 2013 at 22h15m UT. The geocentric radiant position from double-station video observations of the 2013 SPE outburst was determined by Gajdoš et al. (2014) at $\mathrm{RA}=47.6^{\circ}$ and $\mathrm{Dec}=39.4^{\circ}\left(v_{\mathrm{G}}=64.3 \mathrm{kms}^{-1}\right)$ and by Madiedo et al. (2018) at $\mathrm{RA}=47.7^{\circ}$ and $\operatorname{Dec}=39.6^{\circ}\left(v_{\mathrm{G}}=64.6 \mathrm{kms}^{-1}\right)$. The corresponding parameters of the heliocentric orbit are $(q, e$, $i)=\left(0.714 \mathrm{AU}, 0.959,139.1^{\circ}\right)($ Gajdoš et al. 2014) or (0.723 AU, $\left.0.979,139.15^{\circ}\right)($ Madiedo et al. 2018). 
Physical properties of SPE meteors have only been studied by Madiedo et al. (2018) using a sample of video meteors of brightnesses ranging from 1.8 to -6.4 absolute peak magnitude. They studied beginning and terminal heights and found that beginning height increases with increasing meteoroid mass and terminal height decreases with increasing meteoroid mass, which has been found earlier for other meteor showers with a cometary origin (see e.g., Koten et al. 2004). They calculated the so-called $K_{B}$ parameter (Ceplecha 1967) and on the basis of the average value $6.9 \pm 0.2$ analyzed their SPE meteoroids as belonging to the $\mathrm{C}$-group of regular cometary material (Ceplecha 1988). They also studied the strength of SPE meteoroids on the basis of maximum aerodynamic pressure suffered by SPE meteoroids before fragmentation, which has provided a lower limit for their tensile strength. They obtained a maximum aerodynamic pressure of $0.029 \pm 0.003 \mathrm{MPa}$, which is higher than the tensile strength found for Quadrantid and Perseid meteoroids and lower than for Taurids (Trigo-Rodríguez \& Llorca 2006, 2007). They also presented two spectra and analyzed the relative intensity of the Na I-1, Mg I-2, and Fe I-15 multiplets. They concluded that both spectra fit the expected relative intensity for chondritic meteoroids for a meteor velocity of $\sim 65 \mathrm{kms}^{-1}$ and can be considered as normal according to the classification given by Borovička et al. (2005).

Observation of 25 multistation SPE fireballs in the period of 2013-2017 enables us to study physical properties and orbital origin of the shower thanks to determination of atmospheric trajectories, light curves, and heliocentric orbits. We describe our observations and data processing in Sect. 2; activity of fireballs in 2013 and atmospheric trajectories in Sect. 3; physical properties and spectrum in Sect. 4; persistent trains in Sect. 5; and discuss radiant and orbital distribution in Sect. 6.

\section{Instrumentation, observations, and data processing}

The multistation photographic observation of fireballs using fireball networks represents a very efficient and precise method of recording the atmospheric interactions of larger meteoroids. From the short interval of meteoroid ablation, we can determine their atmospheric trajectories, orbits, light curves, and basic physical properties. One of the most advanced operational fireball network is the Czech part of the European Fireball Network. During the outburst in 2013, each station was equipped with a completely Autonomous Fireball Observatory (AFO; Spurný et al. 2007). The AFO imaging system consisted of a Zeiss Distagon fish-eye objective $(f / 3.5, f=30 \mathrm{~mm})$ and a large-format sheet film. All AFOs were equipped with a rotating shutter close to the focal plane to determine fireball velocity. The typical precision of measurement of any individual point on the luminous atmospheric trajectory for fireballs up to approximately $200 \mathrm{~km}$ distance from the stations is about $10-20 \mathrm{~m}$. This precision proportionally decreases with the distance of fireballs from stations. In some ideal cases we can reliably determine fireballs at a distance of about $500 \mathrm{~km}$ from our territory. It enables us to observe fireballs over large parts of Central Europe. Except direct fireball imaging, each AFO also includes an all-sky brightness sensor (radiometer) with a sampling rate of 5000 measurements per second. Therefore, along with the accurate time of fireball passage and its duration, we also obtained a very detailed light curve. These sensors reliably work even under a cloudy sky, so we have basic information about fireball luminosity and its very approximate location even without photographic records.
This system was in full operation during the 2013 SPE enhanced activity, and in parallel the new generation of Digital Autonomous Fireball Observatories (DAFO) was tested at Ondřejov at that time. Over the course of two hours on the evening of September 9, 2013 a total of 19 bright fireballs were recorded. One SPE fireball was also observed on September 6, 2013 and its atmospheric trajectory and heliocentric orbit are also presented. The entire data set includes 12 multistation photographic fireballs and 7 fireballs recorded photographically only from one station. Twelve fireballs were long and bright enough to be recorded photographically from more than one station so that all important parameters describing their atmospheric trajectories, velocities, heliocentric orbits, and basic physical properties could be determined.

DAFO cameras were gradually installed on all Czech stations in 2014 (no SPE was observed that year). These digital versions of AFO (for more details see Spurný et al. 2017) were in operation in the following years and successfully detected two bright SPE fireballs in the morning hours of September 14 and 18 in 2015, six SPE fireballs from September 8 to 9 in 2016, and four SPE fireballs from September 8 to 9 in 2017. Since DAFO takes 35-second-long exposures, the evolution of persistent trains of both 2015 fireballs was possible to study.

We should mention here restrictions specified by the IAU definition from April 2017: "A meteor brighter than absolute visual magnitude (distance of $100 \mathrm{~km}$ ) - 4 is also termed a bolide or a fireball." Since DAFOs are more sensitive than AFOs, it is possible, under ideal circumstances of weather, distance, and angular speed of a meteor, to record meteors fainter than -4 absolute magnitude by our fireball observatories. This was the case of meteors SPE12, SPE17, and SPE20, which do not fall into that definition. It would not make sense not to use these data only because of the limits of this definition. The data are still usable because they are recorded by the same instruments as regular fireballs, so we decided to include these meteors that are technically on the border of the IAU definition of fireballs.

One of the authors (LS) has been operating a digital video camera DFK 31 AU03 with Computar 2.9-8.2 mm, F1.0 lens, and glass diffraction grating with 600 grooves $\mathrm{mm}^{-1}$ since the end of 2013. The field of view of this system is about $40^{\circ}$. The bright SPE fireball of September 14, 2015 flew very favorably in the field of view so that the first and the second order of the spectrum was recorded with $7.5 \mathrm{fps}$ and $1024 \times 768$ pixels resolution.

We also operate surveillance internet protocol (IP) video cameras to supplement the fireball network. The main purpose of the cameras is direct imaging of fragments, but the cameras are also useful for fireball velocity measurement due to their four megapixel resolution and frame rate of 20 images per second. These IP cameras recorded one SPE fireball in 2016 and two in 2017. All presented September epsilon Perseid fireballs were measured and analyzed using our standard procedures (Borovička 1990; Borovička et al. 1995; Ceplecha 1987).

\section{SPE fireball enhanced activity in 2013 and atmospheric trajectories}

Over the course of two hours on the evening of September 9, 2013, 19 bright fireballs in total were recorded by our instruments. It is very probable that all of them belonged to SPE, but we decided to evaluate only 12 multistation cases, where the shower assignment could be unambiguously confirmed. The numbers of observed multistation SPE fireballs in ten-minute bins are shown in Fig. 1 and represent the activity profile of 
Table 1. Atmospheric trajectories of SPE fireballs.

\begin{tabular}{|c|c|c|c|c|c|c|c|c|c|c|c|}
\hline Name & Date & $\begin{array}{l}\text { Time } \\
\text { (UT) }\end{array}$ & $\begin{array}{r}h_{\text {beg }} \\
(\mathrm{km})\end{array}$ & $\begin{array}{r}h_{\text {end }} \\
(\mathrm{km})\end{array}$ & $\begin{array}{c}L \\
(\mathrm{~km})\end{array}$ & $\begin{array}{c}z_{\mathrm{R}} \\
(\mathrm{deg})\end{array}$ & $\begin{array}{c}\lambda_{\text {beg }} \\
(\operatorname{deg})\end{array}$ & $\begin{array}{c}\varphi_{\text {beg }} \\
(\mathrm{deg})\end{array}$ & $\begin{array}{c}\lambda_{\text {end }} \\
(\mathrm{deg})\end{array}$ & $\begin{array}{c}\varphi_{\text {end }} \\
\text { (deg) }\end{array}$ & $\Lambda$ \\
\hline PE01 & p. 6,2013 & 0.3 & 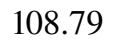 & 9 & 2 THe & . & & 48.780 & 39 & 48.756 & 4 \\
\hline SPE02 & Sep. 9, 2013 & $: 53: 10.7$ & & 87.50 & & & & & & 50.987 & \\
\hline SPE03 & Sep. 9, 2013 & $: 56: 33.6$ & 107.84 & 83.28 & 41.19 & 53.54 & 13.401 & 47.307 & .001 & 47.200 & \\
\hline SPE04 & Sep. 9, 2013 & $: 08: 47.8$ & 101.76 & 81.26 & 30.60 & 48.05 & & 7.531 & 637 & 47.474 & \\
\hline SPE05 & Sep. 9, 2013 & $2: 12: 20.5$ & 119.07 & 77.10 & 66.51 & 51.10 & 12.884 & 49.366 & 12.221 & 49.222 & \\
\hline SPE06 & Sep. 9, 2013 & $2: 13: 29.6$ & 111.59 & 85.88 & 40.27 & 50.48 & 12.325 & 50.865 & & & \\
\hline SPE07 & Sep. 9, 2013 & $: 17: 40.5$ & 102. & 84.00 & 2 & 43 & 22 & 12 & 42 & 85 & \\
\hline SPE08 & Sep. 9, 2013 & 5.4 & 114. & 7 & & & & 43 & & 26 & \\
\hline SPE09 & Sep. 9, 2013 & $: 37: 42.0$ & 108.82 & 75.87 & & & & & & 317 & \\
\hline SPE10 & Sep. 9, 2013 & $22: 40: 51.7$ & 98.64 & 81.18 & 23.41 & 41.84 & & 50.093 & & 50.072 & \\
\hline SPE11 & Sep. 9, 2013 & $2: 58: 19.3$ & 104. & 86.09 & 24.4 & 41 & 15 & 52.176 & & 52.160 & \\
\hline SPE12 & Sep. 9, 2013 & $: 40: 39.6$ & 107.00 & 92.35 & 18.9 & & & 50.328 & & & \\
\hline SPE13 & Sep. 9, 2013 & $: 43: 54.7$ & 106.07 & 85.49 & 25.57 & 36.50 & 12. & 49.362 & 79 & 49.353 & \\
\hline SPE14 & Sep. 14, 2015 & $02: 46: 15.3$ & 130.96 & 77.00 & 55.03 & 11.35 & 12.195 & 49.597 & 12.143 & 49.686 & \\
\hline SPE15 & Sep. 18, 2015 & $00: 13: 44.3$ & 131.86 & 63.62 & 78.53 & 29.84 & 16.955 & 49.066 & 16.431 & 49.075 & \\
\hline SPE16 & Sep & & & & & & & & & & \\
\hline SPE17 & Sep. & $23: 40: 00.5$ & 107.28 & 91 & 18.66 & 34.80 & 16.646 & 60 & 01 & 49.657 & \\
\hline SPE18 & Sep. 9, 2016 & 01:49:09.6 & 106.82 & 85.51 & 21.81 & 12.37 & 19.849 & & & 48.836 & \\
\hline SPE19 & Sep. 9, 2016 & $23: 06: 59.2$ & 117.96 & 73.93 & 58.79 & 41.67 & 14.123 & 49.910 & 13.595 & 49.859 & \\
\hline SPE20 & Sep. 9, 2016 & $23: 09: 56.5$ & 105.29 & 88. & 22.24 & & & & & 50.471 & \\
\hline SPE21 & Sep. 9, 2016 & $23: 26: 10.6$ & 105.80 & 81.04 & 30.44 & 35.66 & 19.194 & 47.061 & 18.965 & 47.045 & \\
\hline SPE22 & Sep. 8, 2017 & $22: 34: 04.6$ & 109.18 & 90.94 & 25.70 & 44.87 & 16.665 & 49.106 & 16.427 & 49.070 & \\
\hline SPE23 & Sep. 8, 2017 & $23: 43: 45.8$ & 111.75 & 83.91 & 33.97 & 35.04 & 14.818 & 49.143 & 14.555 & 49.135 & \\
\hline SPE24 & Sep. 9, 2017 & 01:29:41.0 & 108.82 & 79.45 & 30.14 & 12.97 & & 48.453 & & 48.489 & \\
\hline SPE25 & Sep. 9, 2017 & $23: 39: 15.5$ & 110.96 & 89.42 & 26.13 & 34.55 & 16.728 & 49.403 & 16.527 & 49.399 & \\
\hline
\end{tabular}

Notes. Time corresponds to the beginning of the fireball taken from radiometers, $h_{\text {beg }}$ is beginning and $h_{\text {end }}$ terminal height determined from AFO or DAFO, $L$ is length of atmospheric trajectory, $z_{\mathrm{R}}$ is zenith distance of radiant at terminal point, $\lambda$ and $\varphi$ are the geographical coordinates, and $N$ is number of stations used for determination of the atmospheric trajectory.

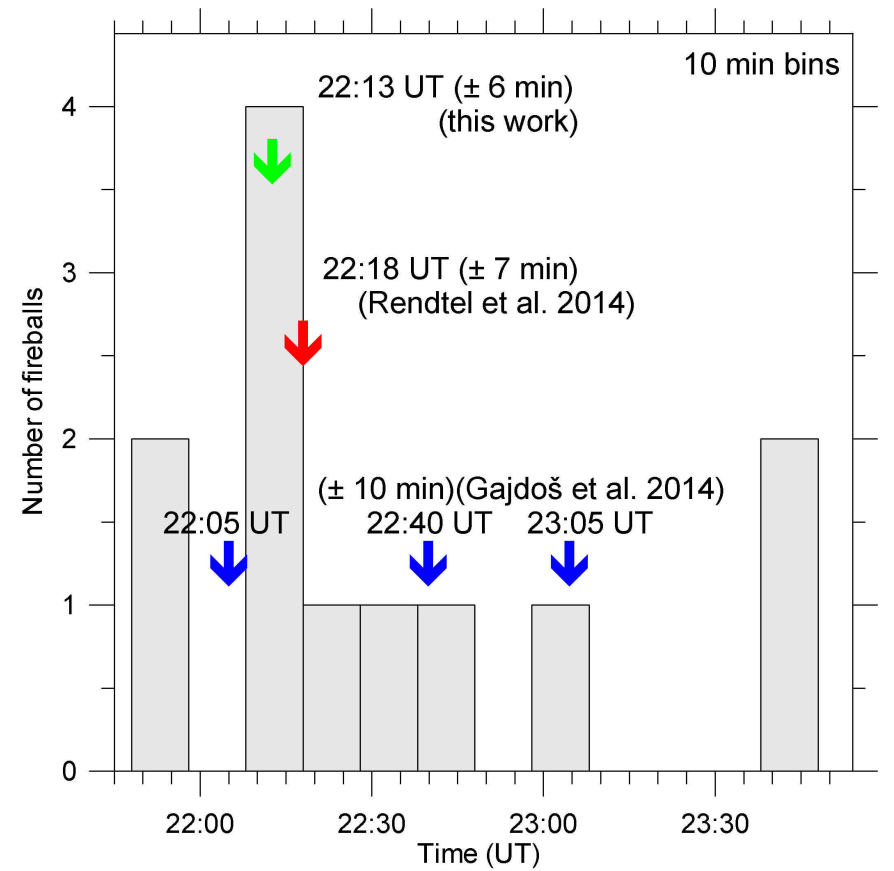

Fig. 1. Number of multistation SPE fireballs in 2013.

SPE 2013 fireballs. We changed the time of the beginning of the ten-minute bins by one minute, which resulted in nine different histograms. The maximum activity was observed from 22:09 to 22:17 UT on the basis of these nine histograms (with a mean value 22:13 UT $\pm 6 \mathrm{~min}$, solar longitude $167.196 \pm 0.004 \mathrm{deg}$ ). Figure 1 shows the histogram, where the maximum activity corresponds to the mean value at 22:13 UT. The maximum activity corresponds to the maximum observed by Rendtel et al. (2014) (for single-station video meteors) and the first maximum of Gajdoš et al. (2014; for double-station video meteors). This comparison therefore shows that the maximum activity of smaller particles (video meteors) occurred at the same time as for larger particles (fireballs).

Atmospheric trajectories are presented in Table 1. Because SPE meteors are very fast and fragile, their atmospheric trajectories are often very short, which can decrease the precision in determination of important parameters, especially the speed. However, in most cases the fireballs listed in Table 1 were recorded from more than two stations, which significantly increases the reliability of the data. The beginning heights range from 99 to $132 \mathrm{~km}$ and approximately rise with initial mass of the meteoroid (Fig. 2). The terminal heights range from 64 to $92 \mathrm{~km}$ and generally decrease with initial mass (Fig. 2). Almost the same dependencies were described by Madiedo et al. (2018) for video meteors. The linear dependencies in Fig. 2 were fit only to 2013 SPE fireballs, which were recorded by photographic film cameras. These dependencies are also satisfied for the majority of SPE fireballs from DAFO cameras, except the beginning heights of the 2015 SPE fireballs. These two fireballs are the brightest SPE, moreover they were close to stations, and thus sensitive DAFOs were about to record these fireballs above $130 \mathrm{~km}$ of altitude, which has never been observed with AFO data for any fireball. If we plot heights, where the SPE14 


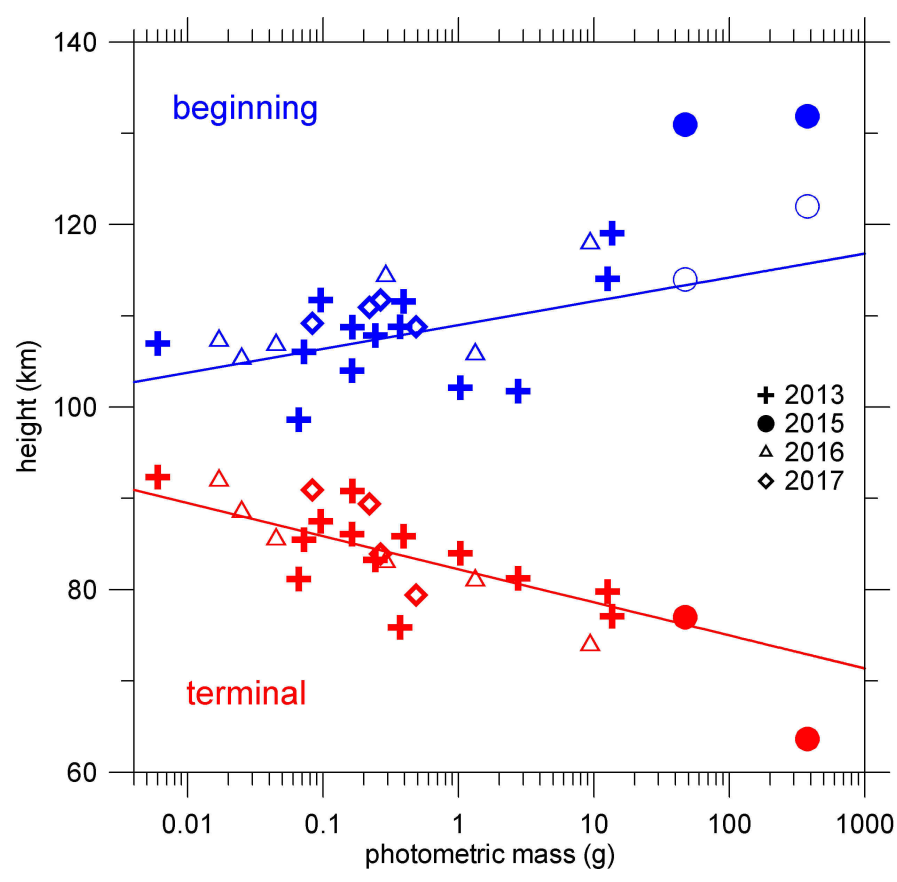

Fig. 2. Beginning and terminal heights as a function of initial photometric mass. The linear dependencies were fit only to 2013 SPE fireballs. Unfilled blue circles indicate heights where SPE14 and SPE15 reached brightnesses of -2 visual magnitudes.

and SPE15 reached a brightness of -2 magnitude (unfilled blue circles in Fig. 2), which approximately corresponds to AFO sensitivity, the dependency fits better.

\section{Physical properties}

The material of meteoroids can be described according to its ablation ability, tensile strength, and chemical composition. Empirical end-height criterion, dynamic pressure at the point of the meteoroid fragmentation, light curve, and meteor spectrum are usually used for these purposes.

\subsection{PE coefficient}

The empirical end-height criterion defined by Ceplecha \& McCrosky (1976) converts terminal height, initial mass and velocity, and slope of the atmospheric trajectory into one PE coefficient describing the fireball ablation ability. Meteoroids are classified into four PE types: I, II, IIIA, and IIIB (Ceplecha 1988). The strongest type I corresponds to stony meteorites; type II to carbonaceous chondrites; type IIIA to regular cometary material; and type IIIB to soft cometary material. The PE coefficients of all SPE fireballs are presented in Table 2. We compared these $P E$ values and their mean value $(P E=-5.31 \pm 0.26)$, which lies near the border between PE types IIIA and II, with other meteor showers (Fig. 3) observed by the same instruments and analyzed by the same procedures. We found Perseids to be the most similar $(P E=-5.27 \pm 0.27)$. Meteoroids of both these showers have almost the same mean value and the same spread of PE coefficients over type II and IIIA (Spurný 1995; Shrbený 2009; Spurný et al. 2014). Orionids are also very similar but contain a large number of IIIB meteoroids, which are interpreted as the softest cometary material, and often exhibit distinct terminal flares (Spurný \& Shrbený 2008; Shrbený 2009). According to PE criterion, the SPE meteoroids are composed
Table 2. Physical properties of SPE fireballs.

\begin{tabular}{lccccccc}
\hline \hline Name & $\begin{array}{c}m_{\text {inf }} \\
(\mathrm{g})\end{array}$ & $\begin{array}{c}M_{\text {abs }} \\
(\mathrm{mag})\end{array}$ & $\begin{array}{c}v_{\text {inf }} \\
\left(\mathrm{km} \mathrm{s}^{-1}\right)\end{array}$ & PE & PE type & $\begin{array}{c}h_{\mathrm{f}} \\
(\mathrm{km})\end{array}$ & $\begin{array}{c}p \\
(\mathrm{MPa})\end{array}$ \\
\hline SPE01 & 1.2 & -5.7 & 65.55 & -5.72 & IIIA/IIIB & - & - \\
SPE02 & 0.7 & -4.7 & 66.01 & -5.25 & II/IIIA & - & - \\
SPE03 & 1.7 & -5.9 & 66.04 & -5.05 & II & - & - \\
SPE04 & 19.6 & -10.2 & 65.81 & -5.40 & IIIA & 88 & 0.0235 \\
SPE05 & 96.4 & -10.9 & 66.07 & -5.36 & IIIA & 88 & 0.0236 \\
SPE06 & 2.8 & -6.4 & 65.75 & -5.39 & IIIA & - & - \\
SPE07 & 7.3 & -9.0 & 65.8 & -5.48 & IIIA & 85.5 & 0.0348 \\
SPE08 & 90.2 & -11.7 & 65.9 & -5.54 & IIIA & 83.5 & 0.0468 \\
SPE09 & 2.6 & -7.8 & 65.8 & -4.69 & II & 80 & 0.0796 \\
SPE10 & 0.5 & -5.6 & 65.9 & -4.77 & II & 85.5 & 0.0349 \\
SPE11 & 1.2 & -5.8 & 65.69 & -5.33 & IIIA & - & - \\
SPE12 & 0.04 & -2.6 & 65.2 & -5.29 & II/IIIA & - & - \\
SPE13 & 0.5 & -5.0 & 66.01 & -5.17 & II & 88 & 0.0236 \\
SPE14 & 334.5 & -12.7 & 65.87 & -5.83 & IIIB & 84.5 & 0.0404 \\
SPE15 & 2670 & -15.0 & 66.44 & -5.35 & IIIA & 81 & 0.0701 \\
SPE16 & 2.1 & -5.8 & 65.59 & -5.05 & II & 89.5 & 0.0185 \\
SPE17 & 0.1 & -3.2 & 65.9 & -5.26 & II/IIIA & - & - \\
SPE18 & 0.3 & -5.0 & 65.8 & -5.20 & II/IIIA & 86 & 0.0322 \\
SPE19 & 66.4 & -11.7 & 65.94 & -5.19 & II/IIIA & 79 & 0.0937 \\
SPE20 & 0.2 & -3.4 & 65.8 & -5.20 & II/IIIA & - & - \\
SPE21 & 9.4 & -10.3 & 66.1 & -5.36 & IIIA & 81.5 & 0.0642 \\
SPE22 & 0.6 & -4.4 & 65.75 & -5.58 & IIIA & - & - \\
SPE23 & 1.9 & -6.7 & 65.84 & -5.30 & II/IIIA & 85 & 0.0374 \\
SPE24 & 3.4 & -6.9 & 65.51 & -5.16 & II & 86 & 0.0319 \\
SPE25 & 1.6 & -6.1 & 65.58 & -5.72 & IIIA/IIIB & - & - \\
\hline & & & & & & &
\end{tabular}

Notes. $m_{\text {inf }}$ is initial photometric mass determined on the basis of luminous efficiency from Revelle \& Ceplecha (2001), $M_{\text {abs }}$ absolute brightness (from $100 \mathrm{~km}$ distance), $v_{\text {inf }}$ initial velocity, PE is explained in Sect. 4.1, $h_{\mathrm{f}}$ is the height where the first significant flare on a light curve was observed, $p$ is dynamic pressure at $h_{\mathrm{f}}$.

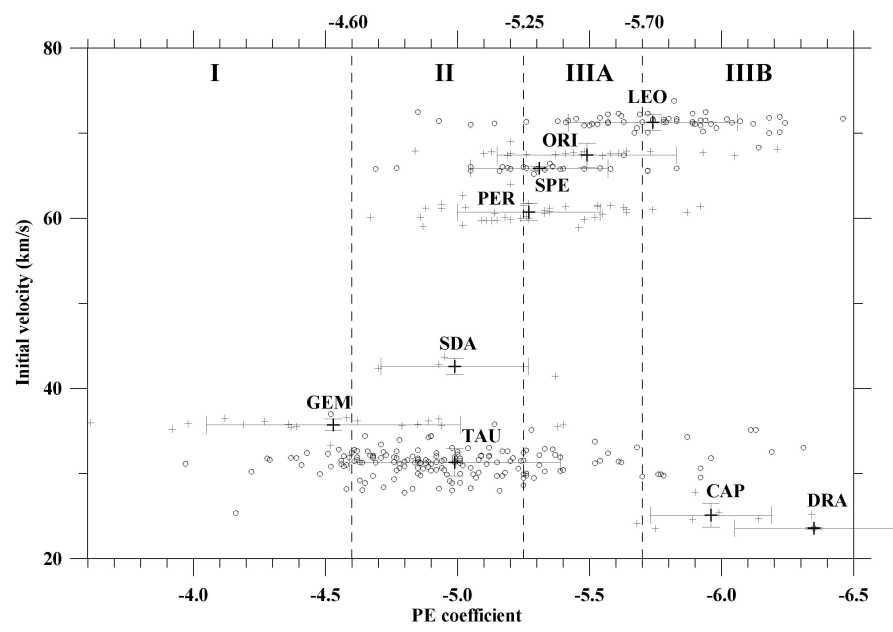

Fig. 3. Dispersion of PE coefficients for some meteor showers. The data were collected from Spurný (1995), Borovička et al. (2007), Spurný \& Shrbený (2008), Shrbený (2009), Shrbený \& Spurný (2009, 2012), Spurný et al. (2014, 2017).

of regular cometary material. The comparison with some other major meteor showers using the PE criterion (Fig. 3) shows that Draconids, $\alpha$-Capricornids, and Leonids are composed on average from more fragile material than SPE, and that Geminids, Taurids, and Southern $\delta$-Aquarids contain stronger material. 


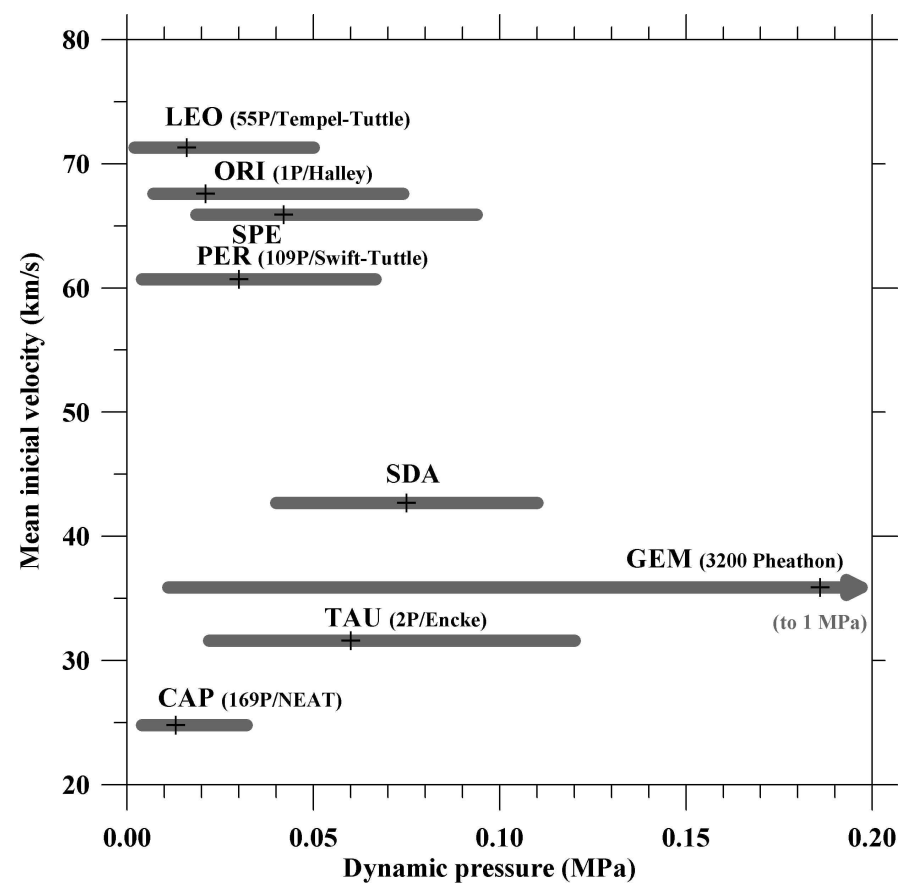

Fig. 4. Dynamic pressures of some major meteor showers. The data were collected from the same sources as in Fig. 3.

\subsection{Dynamic pressures and light curves}

Tensile strength of meteoroid material is usually described by dynamic pressure at the first point of the meteoroid fragmentation. Dynamic pressure is given by the height (i.e., atmospheric density) and velocity just before the fragmentation. We determined the fragmentation heights from light curves, since fragmentation is usually associated with flares (Borovička et al. 2006), which are visible in our detailed radiometric light curves from AFOs and DAFOs. Unfortunately, the height scale of radiometric records had to be determined from photographic records, in some cases as in 2013, only by comparison of the photographic light curve profile and duration. The accuracy of determination of flare heights is about $1 \mathrm{~km}$ in these cases. The accuracy of determination of flare heights from light curve and DAFO data is much better, because of time marks on fireball images produced by electronic shutter. Both radiometer and electronic shutter are continuously corrected by the pulse per second (PPS) pulse of the global positioning system (GPS), so the absolute timing of both records is given with high precision (Spurný et al. 2017). We rounded flare heights (Table 2) to the nearest half $\mathrm{km}$, which better corresponds to the determination of the center of the flare, and not only its maximum brightness. We took the initial velocity as the velocity at fragmentation point, since SPE are very fast meteors and we did not observe any deceleration before these fragmentation points. The derived fragmentation heights and dynamic pressures for fireballs that showed a distinct flare are presented in Table 2 . The mean value of dynamic pressure of 2013 outburst SPE fireballs is $0.038 \pm 0.020 \mathrm{MPa}$ and of all the SPE fireballs $0.044 \pm 0.022 \mathrm{MPa}$. The actual range of dynamic pressures was from 0.0185 to $0.0937 \mathrm{MPa}$. We compared these results with other meteor showers (Fig. 4) and found Orionids and Perseids to be the most similar with almost the same spread of observed dynamic pressures (Shrbený 2009; Spurný \& Shrbený 2008; Spurný et al. 2014), though SPE seem to be slightly stronger and approaching the strength of Taurids.

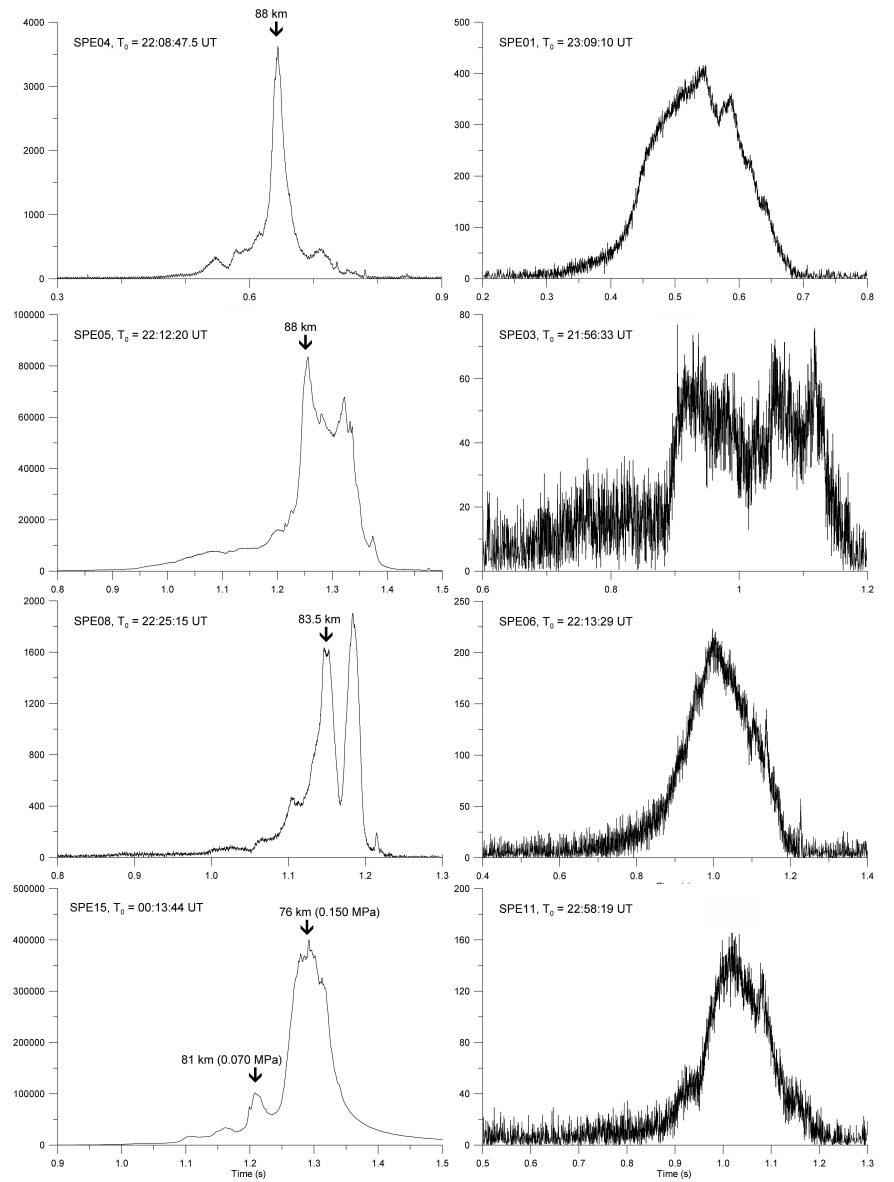

Fig. 5. Examples of radiometric light curves of SPE fireballs. Light curves with distinct flare are on the left, without distinct flare on the right. Vertical axes show intensity in arbitrary units.

Two types of radiometric light curves were observed among the 2013 outburst fireballs and also SPE fireballs in the years 2015-2017. Examples of the light curves are presented in Fig. 5. The first group consists of fireballs exhibiting a distinct flare on the light curve, and thus it was possible to determine the height of fragmentation from the light curve and corresponding dynamic pressure at this point. These fireballs are usually of PE type IIIA or II/IIIA, but also type II fireballs are presented. These fireballs are brighter than -5 to -6 of absolute magnitude. The second group, which has no distinct flares on the light curves, consists of fireballs fainter than -6 of absolute magnitude and also contains fireballs of PE types II to IIIA. The difference between mean brightnesses of both groups is approximately 5 magnitudes, but both groups intersect, so it is unnecessary to specify the fixed difference in brightness. Statistically speaking, the second group has fainter meteors. It seems that SPE meteoroids smaller than approximately $0.1-0.3 \mathrm{~g}$ were not able to produce distinct flares during their ablation, meaning that these small meteoroids are rather compact bodies and do not disintegrate into small grains.

\subsection{Spectrum}

A grating spectrum of the SPE14 was recorded by the DFK 31AU03 charge-coupled device (CCD) video camera with diffraction grating at 600 grooves per $\mathrm{mm}$. The video was taken in progressive scan format with 7.5 frames per second. The spectrum of the fireball was recorded in four individual frames (f9-f12) and the spectrum of the train in two frames (f13 and 

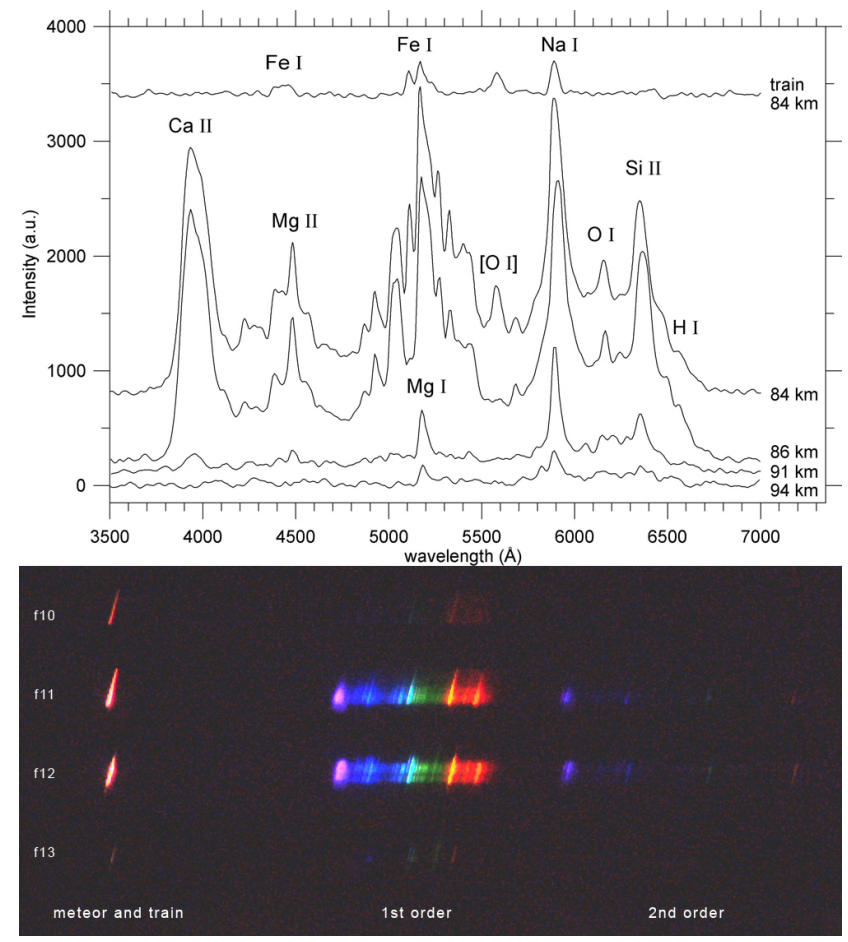

Fig. 6. Spectrum of SPE14. Five individual spectra in different heights are shown in the upper figure and four video frames are shown in the bottom figure (the train is in the $\mathrm{f} 13$ frame).

f14). The first and the last frames show too faint a spectrum to be measured, and thus only four frames were measured (see Fig. 6, bottom panel). Five intensity scans were performed in these four video frames (two in frame f11) to describe the evolution of spectral emission lines and the change of the spectrum by height (see Fig. 6, upper panel). The spectrum was calibrated in wavelength by identification of five lines ( $\mathrm{Na} \mathrm{I}, \mathrm{Mg} \mathrm{I}, \mathrm{Ca}$ II, Mg II, and Si II) typical for such fast meteors. The resolution of the first spectral order was 14 Angstroms per pixel. The identified lines are listed in Table 3.

The spectrum of the fireball was measured at the heights of 94, 91, 86, and $84 \mathrm{~km}$ and the spectrum of its train at $84 \mathrm{~km}$ (heights were determined from the light curve and known atmospheric trajectory; the spectral record itself was not used for the trajectory determination). Intensity scans of individual spectra cover the range of $2 \mathrm{~km}$ in altitude, and thus the above mentioned heights of scans are centers of the measured segments. Only spectral lines of the main (low-temperature) spectrum are presented at $94 \mathrm{~km}$. The second, high-temperature (Borovička 1994), spectrum appears in frame f11 at heights between 90 and $93 \mathrm{~km}$. The first line of the second component visible in the spectrum is Si II - 2 at $93 \mathrm{~km}$, followed by $\mathrm{Mg}$ II -4 at $92 \mathrm{~km}$, and lastly Ca II - 1 at $90 \mathrm{~km}$. The high-temperature component of spectrum was proposed to be connected with a meteor shock wave, which is created at the time when the continuous flow regime forms around the meteoroid, occurring when the molecular mean-free path, $l$, in the plasma around the meteoroid becomes smaller than the meteoroid radius, $r$ (Borovička et al. 2006). According to the computations of Popova (2004), the transition regime from free molecular to continuous flow occurs for a $70 \mathrm{kms}^{-1}$ meteoroid of the size of SPE14 (with a radius of $2 \mathrm{~cm}$ ) between heights of 95 and $92 \mathrm{~km}$ (reflected and evaporated molecules are taken into account for the mean-free path computation). We performed the same computation process described by Bronshten (1983) and Popova (2004) to determine
Table 3. Emission lines identified in the spectrum of SPE14 fireball and its train.

\begin{tabular}{|c|c|c|}
\hline Line & Observed $(\AA)$ & Theoretical $(\AA)$ \\
\hline \multicolumn{3}{|l|}{ Fireball } \\
\hline $\mathrm{Ca}$ I - 1 & 3933 & 3934,3969 \\
\hline Ca I - 2 & 4226 & 4227 \\
\hline $\mathrm{Cr} \mathrm{I}-1$ & 4276 & 4275,4290 \\
\hline Fe I - 42 & 4305 & 4308 \\
\hline $\mathrm{Fe} \mathrm{I}-41$ & 4388 & 4384 \\
\hline Fe I - 2 & 4427 & 4427 \\
\hline Mg II - 4 & 4483 & 4481 \\
\hline $\operatorname{Mg~I}-1$ & 4569 & 4571 \\
\hline Fe I - 318 & 4869 & $4872,4891,4957$ \\
\hline Fe II - 42 & 4926,5019 & 4924,5018 \\
\hline Fe I - 114 & 5048 & 5050 \\
\hline Fe I - 1 & 5112,5163 & $5110,5166,5169$ \\
\hline Mg I - 2 & 5170 & 5173,5184 \\
\hline $\mathrm{Fe} \mathrm{I}-15$ & $5267,5327,5402,5434$ & $5270,5328,5406,5430$ \\
\hline$[\mathrm{O} \mathrm{I}]$ & 5577 & 5577 \\
\hline $\mathrm{Na} I-6$ & 5684 & 5688 \\
\hline $\mathrm{Na} I-1$ & 5889 & 5890,5896 \\
\hline Fe I - 207 & 6070 & 6065 \\
\hline O I - 10 & 6156 & 6157,6158 \\
\hline Fe I - 169 & 6255 & 6253 \\
\hline Si II - 2 & 6349 & 6347,6371 \\
\hline H I - 1 & 6562 & 6563 \\
\hline \multicolumn{3}{|l|}{ Train } \\
\hline $\mathrm{Fe} \mathrm{I}-2$ & $4389,4429,4459$ & $4376,4427,4462$ \\
\hline $\mathrm{Fe} \mathrm{I}-1$ & 5109,5169 & $5110,5166,5169$ \\
\hline$[\mathrm{O} \mathrm{I}]$ & 5579 & 5577 \\
\hline $\mathrm{Na} I-1$ & 5889 & 5890,5896 \\
\hline
\end{tabular}

Notes. Multiplet numbers according to Moore (1945) are given.

the height of the transition regime with the SPE14 atmospheric parameters (and assumed a bulk density of $1 \mathrm{~g} \mathrm{~cm}^{-3}$ ). The transition between the flow regimes can thus be described by the value of the Knudsen number, $K n=1 / r$. The free-molecule flow occurs when $K n>10$. The height depends on the method of determination of the mean-free path. The mean-free path, $l$, of an ideal gas with Maxwell distribution of the velocities of the identical particles is

$l=\frac{k_{\mathrm{B}} T}{\sqrt{2} \pi d^{2} p}$,

where $k_{\mathrm{B}}$ is the Boltzmann constant, $T$ the temperature, $d$ the diameter of particles, and $p$ is the pressure. The mean-free path, $l_{v}$, described by the dynamic viscosity, $\mu$, of gas is

$l_{v}=\frac{\mu}{p} \sqrt{\frac{\pi k_{\mathrm{B}} T}{2 m}}$

where $m$ is the molecular mass. The presence of reflected and evaporated molecules changes the mean-free path, $l$, into $l_{r}$

$l_{r}=6.3 \frac{l}{M a}$,

where $M a$ is the Mach number (about 240 in this case), according to assumptions from Bronshten (1983). The reflected and evaporated molecules do not have to be taken into account 
during the free-molecule flow regime, and thus the value of $K n$ is used for determination of the beginning of the transition regime $(K n>10)$. The reflected and evaporated molecules have to be taken into account during the continuous flow regime, and thus the value of $K n_{\mathrm{r}}=1_{\mathrm{r}} / r$ is used for determination of the end of the transition regime $\left(K n_{\mathrm{r}}<0.1\right)$. We used particles with a molecular diameter of nitrogen $\mathrm{N}_{2}$ of $2.2 \times 10^{-10} \mathrm{~m}$ (Sutton 1965) to determine $K n$. The transition regime may have occurred between 98 and $93 \mathrm{~km}$. If we determine $K n$ by the mean-free path defined by the dynamic viscosity of gas (viscosity according to Dixon 2007) and using the nitrogen $\mathrm{N}_{2}$ molecular mass of $28.0134 \mathrm{u}$ (the unified atomic mass unit $u$ is approximately $1.66 \times 10^{-27} \mathrm{~kg}$ ), the transition regime may occur between 104 and $99 \mathrm{~km}$. We can conclude that the beginning of the continuous flow regime of the SPE14 fireball has occurred under the altitude of $93 \mathrm{~km}$, which corresponds to the observed spectrum.

The forbidden oxygen line [O I] at $557.7 \mathrm{~nm}$ is also present in the SPE14 spectrum (Fig. 6, top panel). It appeared at the height of $87 \mathrm{~km}$, the maximum brightness being at $84 \mathrm{~km}$, and was observed down to $80 \mathrm{~km}$. The appearance of this line corresponds to the fireball maximum brightness and does not correspond to the green train, which was recorded much higher between 120 and $105 \mathrm{~km}$ (too faint to be recorded by this spectral camera) and is mentioned in Sect. 5. Since the [O I] line is present also in the spectrum of the persistent train of SPE14 its creation is probably connected with the creation of the persistent train even if its origin is connected with atmospheric oxygen (Borovička 2006).

Hydrogen line H I-1 at $656.3 \mathrm{~nm}$ is also present in the SPE14 spectrum (Fig. 6, top panel). It appeared in the spectral scan of the $\mathrm{f} 11$ frame, and thus its beginning was between the heights of 85 and $90 \mathrm{~km}$. According to Jenniskens \& Mandell (2004) a significant fraction of the hydrogen atoms in the meteor plasma originated in the meteoroid and if the line is excited in a hot plasma component (high-temperature spectrum) then the H I-1 line is observable. H I-1 line was also observed in Perseids, Geminids, and Leonids (Millman 1963; Jenniskens \& Mandell 2004). The occurrence of hydrogen in meteor spectra may indicate the presence of organic matter or water bound in meteoroid minerals (Borovička \& Jenniskens 2000; Jenniskens \& Mandell 2004). Beech \& Nikolova (2001) estimated the sublimation mass loss rate of water ice of meter- to decameter-sized ice fragments and found the value of $1-0.5 \mathrm{~m}$ in meteoroid radii per orbit for typical meteor-shower-producing comets. Since the size of SPE14 was much smaller it is not probable that the H I-1 line would be caused by the presence of ice in the meteoroid, which is the mechanism proposed by Millman (1963).

The spectrum of the persistent train was recorded at the height of $84 \mathrm{~km}$ and only seven emission lines were identified (Fig. 6): three Fe I-2 lines at 438, 443, and $446 \mathrm{~nm}$; two Fe I-1 lines at 511 and $517 \mathrm{~nm}$; [O I] at $557.7 \mathrm{~nm}$; and Na I-1 at $589 \mathrm{~nm}$. The presence of these lines and the absence of Mg I-2 at $517 \mathrm{~nm}$ corresponds to the emission lines of the afterglow phase of the persistent train (Borovička \& Jenniskens 2000; Borovička 2006). It was not possible to describe the evolution of the spectrum of the train because only one video frame 113 was measurable.

The spectrum of SPE14 is similar to spectra of other shower meteors of similar velocity and brightness. It does not show any exceptional or rare features.

\section{SPE trains in 2015}

Both the 2015 SPE fireballs were recorded inside the Czech part of the EN equipped by DAFOs. DAFO provided digital images
Table 4. Measured points on SPE trains in 2015.

\begin{tabular}{cccccc}
\hline \hline Point & $\begin{array}{c}\text { Height } \\
(\mathrm{km})\end{array}$ & $\begin{array}{c}\text { Duration } \\
(\mathrm{min})\end{array}$ & $\begin{array}{c}\text { Shift } \\
(\mathrm{km})\end{array}$ & $\begin{array}{c}\text { Horizontal speed } \\
\left(\mathrm{m} \mathrm{s}^{-1}\right)\end{array}$ & $\begin{array}{c}\text { Vertical speed } \\
\left(\mathrm{m} \mathrm{s}^{-1}\right)\end{array}$ \\
\hline SPE14 & & & & & \\
\hline A & 91.3 & 34 & 84.4 & $43 \pm 14$ & - \\
C & 89.4 & 9 & 36.2 & $66 \pm 13$ & - \\
B & 87.1 & 9 & 27.7 & $51 \pm 10$ & - \\
D & 84.5 & 1 & - & - & - \\
\hline SPE15 & & & & & \\
\hline A & $93.9 \searrow 90.6$ & 3.2 & 13.8 & $71 \pm 16$ & $15 \pm 13$ \\
B & $88.3 \nearrow 91.0$ & 3.2 & 9.4 & $46 \pm 9$ & $17 \pm 7$ \\
C & 77.7 & 2.2 & 4.8 & $38 \pm 12$ & - \\
\hline
\end{tabular}

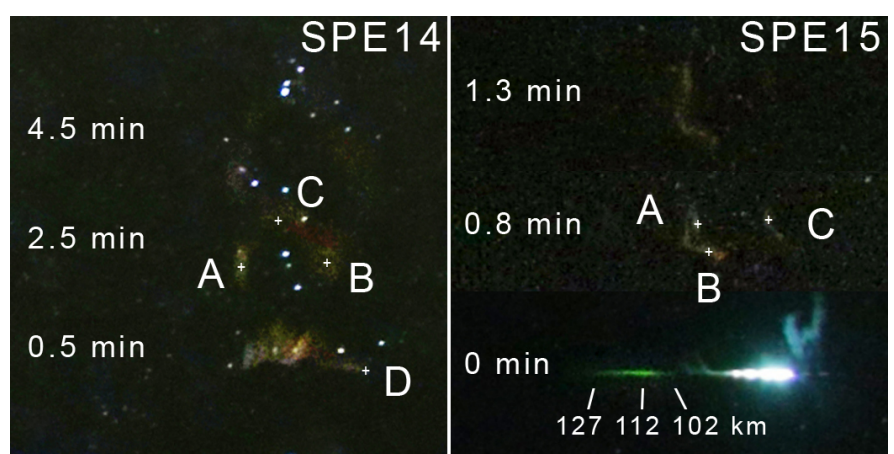

Fig. 7. Trains of the 2015 SPE fireballs. Composition of three individual images of persistent trains recorded in different times is shown for the SPE14 and the SPE15. The times shown mean minutes after the fireballs passages. White crosses denote points (details on A, B, C, and D in Sect. 5) that were identified and measured from two stations. Both fireballs flew from the left to the right.

of $35 \mathrm{~s}$ exposure every $30 \mathrm{~s}$ using two digital cameras inside each DAFO. Since persistent trains of both these fireballs lasted long enough to be recorded on several subsequent images and simultaneously were recorded by the two nearest stations, it was possible to calculate their absolute position and temporal evolution, and estimate high-altitude wind (Table 4).

Four different points were measured on the SPE14 train (see Fig. 7, left) in the range of altitudes from 83 to $91 \mathrm{~km}$. The persistent train of this fireball had a horseshoe-like shape (point A is the beginning, $\mathrm{B}$ the end of the horseshoe, and $\mathrm{C}$ its extreme) in the first nine minutes after the fireball passage. Its part A was possible to measure from two stations for $34 \mathrm{~min}$. Part D (which is a linear shape from 86 down to $83 \mathrm{~km}$ with maximum brightness at $84.5 \mathrm{~km}$ ) of the train disappeared within one minute after the fireball passage and corresponds to the point of fireball maximum brightness. Parts A, B, and C (having heights 91, 87, and $89 \mathrm{~km}$, respectively) correspond to the beginning of the maximum brightness peak and their evolution describes evolution of the persistent train. The train remained in the same heights within the observed period but was shifted northward due to high-altitude wind. Part A was shifted by $84 \mathrm{~km}$ (in $34 \mathrm{~min}$ ) in the direction corresponding to the range of the geographical azimuths from $17^{\circ}$ to $29^{\circ}$. The corresponding mean speed was $43 \mathrm{~ms}^{-1}$. Part B was drifted by $28 \mathrm{~km}$ (in nine minutes) in the azimuth of $348^{\circ}$ with the corresponding mean speed of $51 \mathrm{~ms}^{-1}$, and part $\mathrm{C}$ was drifted by $36 \mathrm{~km}$ (nine minutes) in the azimuth of $7^{\circ}$ with the corresponding mean speed of $66 \mathrm{~ms}^{-1}$ (Table 4). The persistent train of SPE14 fireball was visible on the images 
Table 5. Geocentric radiants and orbital elements of SPE fireballs.

\begin{tabular}{|c|c|c|c|c|c|c|c|c|}
\hline Name & $\begin{array}{c}\alpha_{\mathrm{G}} \\
(\mathrm{deg})\end{array}$ & $\begin{array}{c}\delta_{\mathrm{G}} \\
(\mathrm{deg})\end{array}$ & $\begin{array}{c}\mathrm{v}_{\mathrm{G}} \\
\left(\mathrm{km} \mathrm{s}^{-1}\right)\end{array}$ & $e$ & $\begin{array}{c}q \\
(\mathrm{AU})\end{array}$ & $\begin{array}{c}\omega \\
\text { (deg) }\end{array}$ & $\begin{array}{c}\Omega \\
(\operatorname{deg})\end{array}$ & $\begin{array}{c}i \\
\text { (deg) }\end{array}$ \\
\hline SPE01 & $44.25 \pm 0.10$ & $39.42 \pm 0.03$ & $64.40 \pm 0.10$ & $0.976 \pm 0.007$ & $0.7292 \pm 0.0019$ & $243.9 \pm 0.3$ & 164.321 & $138.06 \pm 0.09$ \\
\hline SPE02 & $47.59 \pm 0.07$ & $39.56 \pm 0.07$ & $64.85 \pm 0.11$ & $0.995 \pm 0.007$ & $0.7244 \pm 0.0020$ & $244.1 \pm 0.4$ & 167.184 & $139.26 \pm 0.13$ \\
\hline SPE03 & $47.45 \pm 0.23$ & $39.56 \pm 0.04$ & $64.9 \pm 0.3$ & $0.999 \pm 0.018$ & $0.723 \pm 0.005$ & $244.2 \pm 0.8$ & 167.186 & $139.16 \pm 0.22$ \\
\hline SPE04 & $47.50 \pm 0.19$ & $39.45 \pm 0.27$ & $64.63 \pm 0.13$ & $0.983 \pm 0.010$ & $0.718 \pm 0.005$ & $245.1 \pm 0.7$ & 167.194 & $139.2 \pm 0.4$ \\
\hline SPE05 & $47.711 \pm 0.015$ & $39.463 \pm 0.010$ & $64.90 \pm 0.05$ & $0.996 \pm 0.003$ & $0.7249 \pm 0.0008$ & $243.99 \pm 0.16$ & 167.197 & $139.50 \pm 0.03$ \\
\hline SPE06 & $47.64 \pm 0.04$ & $39.64 \pm 0.09$ & $64.59 \pm 0.07$ & $0.979 \pm 0.005$ & $0.7226 \pm 0.0017$ & $244.6 \pm 0.3$ & 167.197 & $139.03 \pm 0.13$ \\
\hline SPE07 & $47.38 \pm 0.30$ & $39.64 \pm 0.07$ & $64.6 \pm 0.4$ & $0.987 \pm 0.024$ & $0.720 \pm 0.006$ & $244.8 \pm 1.1$ & 167.200 & $138.9 \pm 0.3$ \\
\hline SPE08 & $47.49 \pm 0.25$ & $39.53 \pm 0.15$ & $64.7 \pm 0.3$ & $0.990 \pm 0.023$ & $0.721 \pm 0.006$ & $244.6 \pm 1.1$ & 167.205 & $139.2 \pm 0.3$ \\
\hline SPE09 & $47.48 \pm 0.21$ & $39.29 \pm 0.17$ & $64.6 \pm 0.4$ & $0.982 \pm 0.023$ & $0.715 \pm 0.006$ & $245.5 \pm 1.1$ & 167.214 & $139.5 \pm 0.3$ \\
\hline SPE10 & $47.47 \pm 0.13$ & $39.51 \pm 0.12$ & $64.7 \pm 0.6$ & $0.99 \pm 0.04$ & $0.720 \pm 0.009$ & $244.7 \pm 1.8$ & 167.216 & $139.2 \pm 0.4$ \\
\hline SPE11 & $47.64 \pm 0.22$ & $39.44 \pm 0.36$ & $64.54 \pm 0.26$ & $0.975 \pm 0.017$ & $0.718 \pm 0.007$ & $245.2 \pm 1.1$ & 167.228 & $139.3 \pm 0.6$ \\
\hline SPE12 & $47.73 \pm 0.10$ & $39.40 \pm 0.09$ & $64.1 \pm 1.4$ & $0.95 \pm 0.09$ & $0.712 \pm 0.021$ & $246.6 \pm 4.4$ & 167.256 & $139.2 \pm 0.7$ \\
\hline SPE13 & $47.64 \pm 0.03$ & $39.529 \pm 0.013$ & $64.88 \pm 0.13$ & $0.998 \pm 0.009$ & $0.7235 \pm 0.0018$ & $244.1 \pm 0.4$ & 58 & $139.32 \pm 0.07$ \\
\hline SPE14 & $51.120 \pm 0.026$ & $38.851 \pm 0.010$ & $64.91 \pm 0.07$ & $0.992 \pm 0.005$ & $0.6906 \pm 0.0011$ & $248.28 \pm 0.23$ & 170.773 & $141.30 \pm 0.04$ \\
\hline SPE15 & $56.945 \pm 0.018$ & $40.331 \pm 0.013$ & $65.35 \pm 0.07$ & $0.999 \pm 0.005$ & $0.7213 \pm 0.0010$ & $244.20 \pm 0.20$ & 174.570 & $141.41 \pm 0.04$ \\
\hline SPE16 & $46.51 \pm 0.07$ & $39.31 \pm 0.12$ & $64.41 \pm 0.13$ & $0.972 \pm 0.009$ & $0.716 \pm 0.003$ & $245.6 \pm 0.5$ & 166.413 & $138.98 \pm 0.19$ \\
\hline SPE17 & $47.41 \pm 0.15$ & $39.42 \pm 0.06$ & $64.7 \pm 0.6$ & $0.98 \pm 0.04$ & $0.732 \pm 0.009$ & $243.5 \pm 1.9$ & 166.506 & $139.6 \pm 0.3$ \\
\hline SPE18 & $47.28 \pm 0.13$ & $39.92 \pm 0.05$ & $64.8 \pm 0.4$ & $0.99 \pm 0.03$ & $0.737 \pm 0.006$ & $242.5 \pm 1.2$ & 166.593 & $138.73 \pm 0.24$ \\
\hline SPE19 & $47.956 \pm 0.014$ & $39.328 \pm 0.015$ & $64.80 \pm 0.05$ & $0.988 \pm 0.003$ & $0.7197 \pm 0.0008$ & $244.77 \pm 0.15$ & 167.455 & $139.73 \pm 0.03$ \\
\hline SPE20 & $47.91 \pm 0.13$ & $39.37 \pm 0.11$ & $64.7 \pm 0.4$ & $0.981 \pm 0.023$ & $0.718 \pm 0.006$ & $245.1 \pm 1.1$ & 167.457 & $139.58 \pm 0.25$ \\
\hline SPE21 & $48.50 \pm 0.26$ & $39.52 \pm 0.17$ & $65.0 \pm 0.4$ & $0.993 \pm 0.025$ & $0.732 \pm 0.006$ & $243.2 \pm 1.2$ & 167.468 & $139.9 \pm 0.4$ \\
\hline SPE22 & $46.57 \pm 0.13$ & $39.80 \pm 0.12$ & $64.59 \pm 0.26$ & $0.984 \pm 0.017$ & $0.731 \pm 0.004$ & $243.5 \pm 0.8$ & 166.214 & $138.48 \pm 0.20$ \\
\hline SPE23 & $46.63 \pm 0.03$ & $39.593 \pm 0.021$ & $64.72 \pm 0.14$ & $0.989 \pm 0.009$ & $0.7292 \pm 0.0019$ & $243.6 \pm 0.4$ & 166.261 & $138.87 \pm 0.08$ \\
\hline SPE24 & $46.42 \pm 0.10$ & $39.64 \pm 0.13$ & $64.51 \pm 0.18$ & $0.982 \pm 0.012$ & $0.723 \pm 0.003$ & $244.5 \pm 0.6$ & 166.332 & $138.53 \pm 0.23$ \\
\hline SPE25 & $47.67 \pm 0.05$ & $39.53 \pm 0.08$ & $64.46 \pm 0.10$ & $0.970 \pm 0.006$ & $0.7193 \pm 0.0020$ & $245.2 \pm 0.4$ & 167.229 & $139.17 \pm 0.14$ \\
\hline 2013 & $47.67 \pm 0.04$ & $39.493 \pm 0.013$ & $64.79 \pm 0.06$ & $0.991 \pm 0.003$ & $0.7240 \pm 0.0013$ & $244.20 \pm 0.21$ & $167.211 \pm 0.025$ & $139.43 \pm 0.07$ \\
\hline 2013-G & $47.6 \pm 0.7$ & $39.4 \pm 0.5$ & 64.3 & 0.96 & 0.714 & 246 & 167.2 & 139.1 \\
\hline 2013-M & $47.73 \pm 0.20$ & $39.60 \pm 0.18$ & 64.62 & 0.979 & 0.723 & 244.4 & 167.20 & 139.15 \\
\hline MDC orbit & 47.4 & 39.0 & 65.6 & 1.016 & 0.734 & 242.4 & 166.9 & 140.6 \\
\hline
\end{tabular}

Notes. "2013" indicate weighted mean parameters and standard deviations determined for all SPE 2013 outburst fireballs. MDC orbit is based on three photographic fireballs from the IAU Meteor Data Center list of photographic orbits (Gajdoš \& Porubčan 2005). 2013-G and 2013-M are mean parameters of 2013 SPE by Gajdoš et al. (2014) and Madiedo et al. (2018), respectively. Values of individual entries are given with an accuracy of one last digit or with one standard deviation.

about $45 \mathrm{~min}$ from the nearest station. An increase of brightness of the SPE14 persistent train in its continuum phase, probably due to exothermic chemical reactions, was observed one, eight, and eleven minutes after the fireball passage only in part A.

Three different points were measured on the SPE15 train (see Fig. 7, right) in the range of altitudes from 77 to $93 \mathrm{~km}$. The persistent train of this fireball had a very complicated shape. Two diffuse clouds (points A and B) were possible to measure from two stations for less than $3.5 \mathrm{~min}$ (visible on the images for six minutes from the nearest station). Part $\mathrm{C}$ disappeared within two minutes after the fireball passage and corresponds to the fireball peak brightness. It remained at between 77 and $78 \mathrm{~km}$ of altitude. On the other hand, unlike SPE14, parts A and B showed vertical evolution. Part A moved from $93 \mathrm{~km}$ down to $91 \mathrm{~km}$, and part B moved from 88 up to $91 \mathrm{~km}$. Also horizontal shift differs for individual parts. Parts $\mathrm{A}$ and $\mathrm{C}$ were both shifted approximately to the north (part $\mathrm{A}$ in the azimuth of $331^{\circ}$ and part $\mathrm{C}$ of $0^{\circ}$ ), however part $\mathrm{B}$, located between them, was shifted approximately to the west $\left(274^{\circ}\right)$. Mean horizontal drift speeds were $71 \mathrm{~ms}^{-1}$ for part A, $46 \mathrm{~ms}^{-1}$ for part B, and $38 \mathrm{~ms}^{-1}$ for part C. Mean vertical drift speeds were $15 \mathrm{~ms}^{-1}$ (downward) for part $\mathrm{A}$, and $17 \mathrm{~ms}^{-1}$ (upward) for part B (Table 4).

Thanks to a lucky beginning of the exposure of one digital camera, which occurred at the time when the SPE15 fireball was in the middle of its trajectory, the short duration train emitting a forbidden line of [O I] at $557.7 \mathrm{~nm}$ (Halliday 1958) was recorded (Fig. 7, right) in the heights from 127 down to $102 \mathrm{~km}$ with a maximum brightness at $112 \mathrm{~km}$. The short duration train was captured $0.69 \mathrm{~s}$ after its creation in the height of $127 \mathrm{~km}$ and $0.25 \mathrm{~s}$ after its creation in the height of $102 \mathrm{~km}$. Since the maximum intensity of this green train is reached about $0.1 \mathrm{~s}$ after its creation (Borovička 2006) the height of the brightest part observed does not have to correspond to the real height of maximum intensity and can be shifted a little upward. However, the image of the fireball where the green train is exposed together with the rest of the fireball, confirms the heights mentioned above (heights for SPE14 are from 120 down to $105 \mathrm{~km}$ with a maximum at approximately $112 \mathrm{~km}$ using this approach, which does not correspond to heights where the forbidden oxygen line [O I] at $557.7 \mathrm{~nm}$ was observed in spectrum of SPE14; see Sect. 4.3). An increase of brightness of the SPE15 persistent train in its continuum phase due to exothermic chemical reactions was observed $3.5 \mathrm{~min}$ after the fireball passage. This occured only in part B whose height was $91 \mathrm{~km}$ at that time.

\section{Radiant and orbit}

Geocentric radiant positions and orbital elements for all SPE fireballs are listed in Table 5. All values are given in the J2000.0 equinox.

We determined the dependency of RA $\alpha_{\mathrm{G}}$, of geocentric radiants on solar longitude, $\lambda_{\odot}$ (Fig. 8). The linear fit 


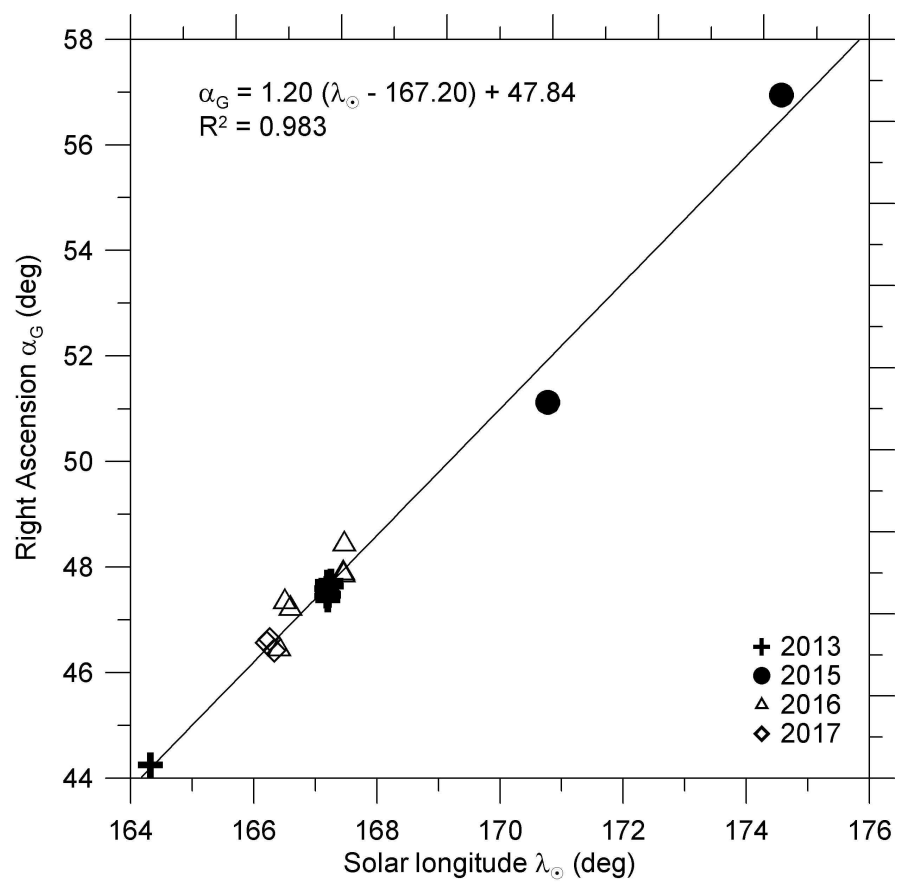

Fig. 8. Geocentric Right Ascension of all SPE fireballs as a function of solar longitude.

$\alpha_{\mathrm{G}}=1.20\left(\lambda_{\odot}-167.20\right)+47.84$ has a coefficient of determination $R^{2}=0.983$. All radiants were used for the fit, which corresponds to SPE fireballs observed within the range of solar longitude from $164^{\circ}$ to $174^{\circ}$ (September 6-18). The derived radiant drift in $\mathrm{RA}$ is $1.2^{\circ}$ per day. Unambiguous drift in Dec was not observed. The corrected radiants are shown in Fig. 9. The mean corrected geocentric radiant for solar longitude $167.20^{\circ}$ determined from 20 fireballs (red symbols in Fig. 9) was computed as the weighted average and has RA $47.67 \pm 0.02^{\circ}$ and Dec $39.47 \pm 0.02^{\circ}$, and is the same as the SPE 2013 outburst radiant within the errors (Table 5). SPE from 2013, 2016, and 2017 are included. Five meteors were not included in the computation of the mean radiant (blue symbols in Fig. 9), since they are well outside the mean radiant area even within the range of their errors. Two of these meteors are both bright SPE fireballs from 2015 and three of these meteors are three of six SPE meteors from 2016. It is possible that the Earth encountered a different trail or a more dispersed part of the trail in 2015-2016 than in 2013 and 2017.

Rendtel et al. (2014) presented a population index of 2013 SPE video meteors observed near the peak of the 2013 outburst. The value $1.45 \pm 0.15$ is the second smallest (after Leonids 1998 published by Arlt 1998) ever observed for meteor streams. Values of population index smaller than 2.0 have been observed only on a few occasions. Most of these cases have been found to be consist of meteoroids trapped in resonances with Jupiter's orbital period, however Rendtel et al. (2014) concluded that the 2013 SPE outburst was caused by very close encounter of the Earth and the center of a SPE trail. On the basis of the work of Lyytinen \& Jenniskens (2003), such shower outbursts from LPCs can only occur from one-revolution trail. We determined the mean radiant position and the mean orbital parameters of the SPE 2013 outburst as the weighted average from all outburst SPE fireballs (Table 5). On the basis of the mean orbit of SPE 2013 outburst fireballs we conclude that the outburst was caused by particles from a LPC on a retrograde orbit, and thus that the outburst was caused by particles from one-revolution trail.

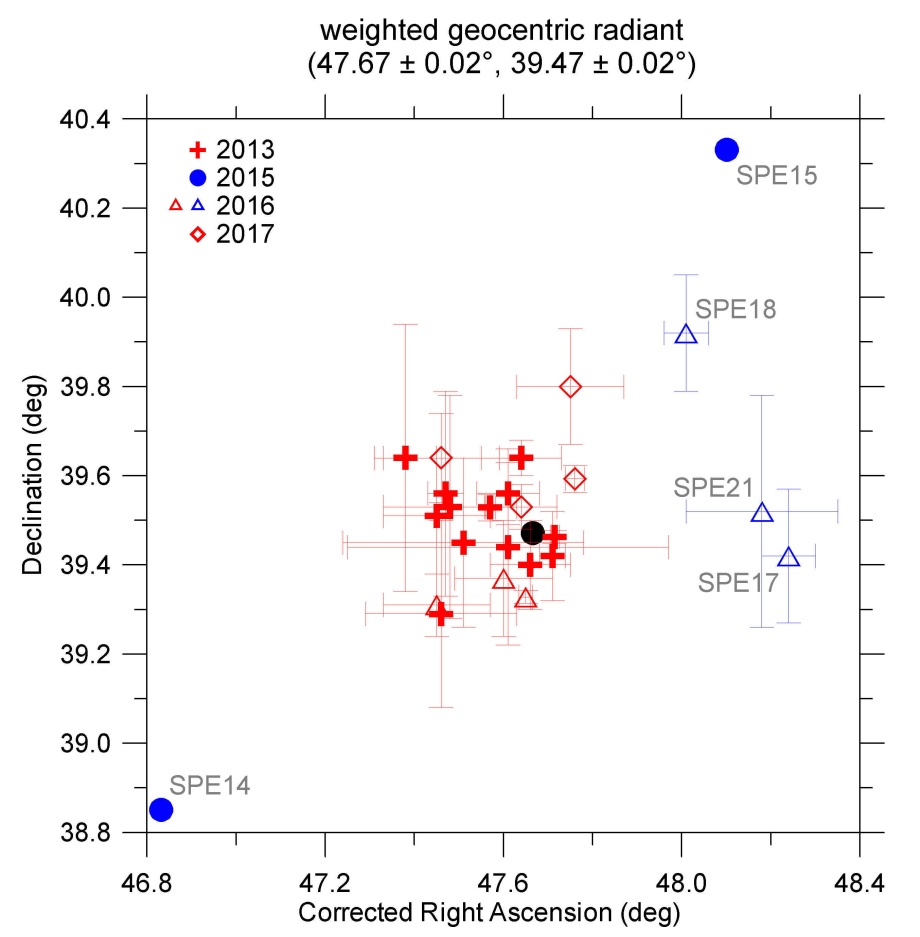

Fig. 9. Geocentric radiants with standard deviations of all SPE fireballs corrected for the radiant drift in right ascension to solar longitude $167.2^{\circ}$. Standard deviation of SPE14 and SPE15 is the same as the size of used symbols (blue circles). The weighted mean radiant (black circle) is determined from 20 radiants (red symbols) and the size of the circle corresponds to the radiant standard deviation.

We take the mean orbital parameters of the SPE 2013 outburst for the best estimation of a parent comet orbital period. The semi-major axis of the mean orbit is $80 \pm 27 \mathrm{AU}$. The orbital period, $P$, ranges from 380 to $1100 \mathrm{yr}$ with the most probable value around $720 \mathrm{yr}$. The most probable orbital periods on the basis of data published by Gajdoš et al. (2014) and Madiedo et al. (2018) were 70 and $200 \mathrm{yr}$, respectively. We conclude that these authors underestimated the eccentricity of the orbit.

It is possible to compare the mean orbit of the SPE 2013 outburst (with an orbital period of $720 \mathrm{yr}$ ) with showers that have known LPCs as a parent. April Lyrids (LYR) with C/1861 G1 Thatcher and Aurigids (AUR) with C/1911 N1 Kiess are two established showers with known parent comets that have an orbital period above $200 \mathrm{yr}$. The orbital period of the comet Thatcher is about $415 \mathrm{yr}$ and the semi-major axis of the mean photographic orbit of nine Lyrid fireballs is $67 \mathrm{AU}$, which is an orbital period of $540 \mathrm{yr}$ (Gajdoš \& Porubčan 2005). The orbital period of the comet Kiess is about $2000 \mathrm{yr}$ and recently, an outburst of Aurigids was observed in 2007 (Rendtel 2007; Jenniskens \& Vaubaillon 2008; Atreya \& Christou 2009). Unfortunately, only five double-station video meteors are available and the accuracy of velocity measurements is not sufficient so it was difficult to compute the semi-major axis accurately. Lyytinen \& Jenniskens (2003) performed modeling of $\alpha$-Monocerotids (AMO), which have unknown parent LPC, and concluded, on the basis of all recent outbursts of the shower, that only the one-revolution particles can explain observed outbursts and their best solution has a semi-major axis $a=75 \pm 5 \mathrm{AU}$ (orbital period $650 \mathrm{yr}$ ). Lyytinen \& Jenniskens (2003) also concluded that strong outbursts are expected only from comets with periods under a few thousand years (except giant comets of HaleBopp-size) because the dust density in one-revolution trail is 
proportional to $a^{-2.5}$ (Sykes et al. 1990). Our mean orbit of the SPE 2013 outburst is in agreement with this conclusion.

\section{Conclusions}

We present results on atmospheric trajectories, orbits, light curves, and physical properties of 25 SPE fireballs recorded by cameras of the Czech Fireball Network from 2013 to 2017. Our data have higher accuracy and reliability than other data presented so far. The main conclusions are as follows:

- The maximum fireball activity of 2013 SPE outburst is at $22: 13 \pm 6$ min UT (solar longitude $167.196 \pm 0.004 \mathrm{deg}$ ) and corresponds to the maximum of video meteors (Rendtel et al. 2014; Gajdoš et al. 2014).

- On the basis of atmospheric trajectories, ablation abilities, and dynamic pressures SPE material is of cometary origin and is a bit harder than that of Orionids and statistically the same as that of Perseids.

- Radiation of meteor shock wave started between altitudes 90-93 km judging from spectral records. Theoretically, the shock wave is expected to develop below the altitude of $93 \mathrm{~km}$.

- The spectrum is similar to spectra of other shower meteors with similar velocity and brightness and does not show any exceptional or rare features.

- Double-station evolution of two SPE persistent trains was determined. The longest duration of the trains was observed at the height of $91 \mathrm{~km}$ in both the cases. High-altitude wind components were estimated from horizontal and vertical shifts of these trains. Horizontal speed ranges from 40 to $70 \mathrm{~ms}^{-1}$ and vertical speed is under $20 \mathrm{~ms}^{-1}$.

- Geocentric radiant of the 2013 SPE outburst for solar longitude $167.20^{\circ}$ is $47.67 \pm 0.04^{\circ} ; 39.493 \pm 0.013^{\circ}$ and can be used for confirmation of future outbursts (in 2026 and 2030), as predicted by Rendtel et al. (2014).

- The parent body of the shower is an unknown LPC on retrograde orbit with an orbital period between 380 and $1100 \mathrm{yr}$; and the 2013 outburst was caused by particles from one-revolution trail.

Acknowledgements. This work was supported by the project RVO:67985815, by Praemium Academiae of the AS ČR, and by GA ČR grant 16-00761S.

\section{References}

Arlt, R. 1998, WGN, J. Int. Meteor Organ., 26, 239

Atreya, P., \& Christou, A. A. 2009, MNRAS, 393, 1493

Beech, M., \& Nikolova, S. 2001, Planet. Space Sci., 49, 23

Borovička, J. 1990, Bull. Astr. Inst. Czechosl., 41, 391

Borovička, J. 1994, Planet. Space Sci., 42, 145
Borovička, J. 2006, J. R. Astron. Soc. Can., 100, 194

Borovička, J., \& Jenniskens, P. 2000, Earth Moon Planets, 82, 399

Borovička, J., Spurný, P., \& Keclíková, J. 1995, A\&AS, 112, 173

Borovička, J., Koten, P., Spurný, P., Boček, J., \& Štork, R. 2005, Icarus, 174, 15

Borovička, J., Weber, M., \& Boček, J. 2006, WGN, J. Int. Meteor Organ., 34, 49 Borovička, J., Spurný, P., \& Koten, P. 2007, A\&A, 473, 661

Bronshten, V. A. 1983, Physics of Meteoric Phenomena (The Netherlands: Springer)

Ceplecha, Z. 1967, Smithson. Contrib. Astrophys., 11, 35

Ceplecha, Z. 1987, Bull. astr. Inst. Czechosl., 38, 222

Ceplecha, Z. 1988, Bull. astr. Inst. Czechosl., 39, 221

Ceplecha, Z., \& McCrosky, R. E. 1976, J. Geophys. Res., 81, 6257

Cook, A. F. 1973, A Working List of Meteor Streams, eds. C. L. Hemenway, P. M. Millman, \& A. F. Cook, Proc. IAU Colloq. 13, 319, 183

Denning, W. F. 1878, MNRAS, 39, 22

Denning, W. F. 1882, The Observatory, 5, 262

Dixon, J. C. 2007, The Shock Absorber Handbook, 2nd edn. (New York: John Wiley \& Sons)

Gajdoš, Š., \& Porubčan, V. 2005, in Dynamics of Populations of Planetary Systems, eds. Z. Knežević \& A. Milani, IAU Colloq. 197, 393

Gajdoš, Š., Tóth, J., Kornoš, L., Koukal, J., \& Piffl, R. 2014, WGN, J. Int. Meteor Organ., 42, 48

Halliday, I. 1958, ApJ, 128, 441

Hoffmeister, C. 1948, Meteorstrome. Meteoric currents. (Leipzig: J.A. Barth)

Jenniskens, P., \& Mandell, A. M. 2004, Astrobiology, 4, 123

Jenniskens, P., \& Vaubaillon, J. 2008, Earth Moon Planets, 102, 157

Jenniskens, P., Brower, J., Martsching, P., et al. 2008, CBET, 1501, 1

Jenniskens, P., Nénon, Q., Albers, J., et al. 2016, Icarus, 266, 331

Koten, P., Borovička, J., Spurný, P., Betlem, H., \& Evans, S. 2004, A\&A, 428, 683

Lyytinen, E., \& Jenniskens, P. 2003, Icarus, 162, 443

Madiedo, J. M., Zamorano, J., Trigo-Rodríguez, J. M., et al. 2018, MNRAS, 480, 2501

Millman, P. M. 1963, Smithson. Contrib. Astrophys., 7, 119

Molau, S., \& Kac, J. 2008, WGN, J. Int. Meteor Organ., 36, 112

Moore, C. E. 1945, Contributions from the Princeton University Observatory (Princeton, N.J.: The Observatory), 21, I

Popova, O. 2004, Earth Moon Planets, 95, 303

Porubčan, V., \& Gavajdová, M. 1994, Planet. Space Sci., 42, 151

Rendtel, J. 1993, in Proceedings of the International Meteor Conference, 11th IMC, Smolenice, Slovakia, 1992, 67

Rendtel, J. 2007, WGN, J. Int. Meteor Organ., 35, 108

Rendtel, J., \& Molau, S. 2010, WGN, J. Int. Meteor Organ., 38, 161

Rendtel, J., Lyytinen, E., Molau, S., \& Barentsen, G. 2014, WGN, J. Int. Meteor Organ., 42, 40

Revelle, D. O., \& Ceplecha, Z. 2001, in Meteoroids 2001 Conference, ed. B. Warmbein, ESA SP, 495, 507

Shrbený, L. 2009, PhD Thesis, Charles University in Prague, Czech Republic

Shrbený, L., \& Spurný, P. 2009, A\&A, 506, 1445

Shrbený, L., \& Spurný, P. 2012, in Asteroids, Comets, Meteors 2012, 1667, 6436

Spurný, P. 1995, Earth Moon Planets, 68, 529

Spurný, P., \& Shrbený, L. 2008, Earth Moon Planets, 102, 141

Spurný, P., Borovička, J., \& Shrbený, L. 2007, IAU Symp., 236, 121

Spurný, P., Shrbený, L., Borovička, J., et al. 2014, A\&A, 563, A64

Spurný, P., Borovička, J., Mucke, H., \& Svoreň, J. 2017, A\&A, 605, A68

Sutton, L. E. 1965, Soil Sci., 100, 76

Sykes, M. V., Lien, D. J., \& Walker, R. G. 1990, Icarus, 86, 236

Trigo-Rodríguez, J. M., \& Llorca, J. 2006, MNRAS, 372, 655

Trigo-Rodríguez, J. M., \& Llorca, J. 2007, MNRAS, 375, 415 